\title{
Evidence for Reduced Experience-Dependent Dendritic Spine Plasticity in the Aging Prefrontal Cortex
}

\author{
Erik B. Bloss, ${ }^{1}$ William G. Janssen, ${ }^{1}$ Daniel T. Ohm, ${ }^{1}$ Frank J. Yuk, ${ }^{1}$ Shannon Wadsworth, ${ }^{1}$ Karl M. Saardi, ${ }^{1}$ \\ Bruce S. McEwen, ${ }^{3}$ and John H. Morrison ${ }^{1,2}$ \\ ${ }^{1}$ Department of Neuroscience and ${ }^{2}$ Department of Geriatrics and Palliative Care, Mount Sinai School of Medicine, New York, New York 10029, and \\ ${ }^{3}$ Laboratory of Neuroendocrinology, The Rockefeller University, New York, New York 10021
}

Cognitive functions that require the prefrontal cortex are highly sensitive to aging in humans, nonhuman primates, and rodents, although the neurobiological correlates of this vulnerability remain largely unknown. It has been proposed that dendritic spines represent the primary site of structural plasticity in the adult brain, and recent data have supported the hypothesis that aging is associated with alterations of dendritic spine morphology and plasticity in prefrontal cortex. However, no study to date has directly examined whether aging alters the capacity for experience-dependent spine plasticity in aging prefrontal neurons. To address this possibility, we used young, middle-aged, and aged rats in a behavioral stress paradigm known to produce spine remodeling in prefrontal cortical neurons. In young rats, stress resulted in dendritic spine loss and altered patterns of spine morphology; in contrast, spines from middle-aged and aged animals were remarkably stable and did not show evidence of remodeling. The loss of stress-induced spine plasticity observed in aging rats occurred alongside robust age-related reductions in spine density and shifts in remaining spine morphology. Together, the data presented here provide the first evidence that experience-dependent spine plasticity is altered by aging in prefrontal cortex, and support a model in which dendritic spines become progressively less plastic in the aging brain.

\section{Introduction}

The prefrontal cortex (PFC) controls a wide range of cognitive processes by integrating information across cortical and subcortical structures to establish and update the "rules of the game" in a dynamic, continuous fashion (Miller, 2000). Performance on tasks that require the $\mathrm{PFC}$ is highly vulnerable to decline with age in humans, nonhuman primates, and rodents (Gallagher and Rapp, 1997; Grady, 2008), although the neurobiological correlates of these deficits remain largely unknown (Hof and Morrison, 2004; Burke and Barnes, 2006).

As axons and dendrites appear to be relatively stable, dendritic spines appear to be the primary site of structural plasticity in the adult brain (Holtmaat and Svoboda, 2009). Spines form the postsynaptic component of excitatory synapses in neocortical circuits and are capable of rapid formation, expansion, contraction, and elimination (Grutzendler et al., 2002; Trachtenberg et al., 2002; Matsuzaki et al., 2004; Okamoto et al., 2004; Zhou et al., 2004; Yasumatsu et al., 2008). Individual spines exhibit a wide, dynamic morphological continuum, the vast majority of spines being characterized by a thin neck and small, thin head (i.e., thin

Received Feb. 16, 2011; revised April 13, 2011; accepted April 18, 2011.

Author contributions: E.B.B., B.S.M., and J.H.M. designed research; E.B.B., W.G.J., D.T.O., F.J.Y., S.W., and K.M.S. performed research; E.B.B. analyzed data; E.B.B., B.S.M., and J.H.M. wrote the paper.

This work is supported by NIA Grant F31AG034794 to E.B.B. and NIHM Grant MH58911 to B.S.M and J.H.M. We thank Drs. Patrick Hof, Dani Dumitriu, Yuko Hara, Russ Romeo, Rebecca Shansky, and Rishi Puri for expert advice and assistance.

The authors declare no competing financial interests.

Correspondence should be addressed to Dr. John H. Morrison, Mount Sinai School of Medicine, Department of Neuroscience, One Gustave L. Levy Place, New York, NY 10029. E-mail: john.morrison@mssm.edu.

DOI:10.1523/JNEUROSCI.0839-11.2011

Copyright $\odot 2011$ the authors $\quad 0270-6474 / 11 / 317831-09 \$ 15.00 / 0$ spines), whereas others have a thin neck and a large, mushroomshaped head (i.e., mushroom spines) or no neck and a stubby appearance (i.e., stubby spines) (Peters and Kaiserman-Abramof, 1970; Harris and Stevens, 1989). Individual spine morphology can predict both spine stability and synaptic strength, as large spines generally persist for weeks to months and form strong synapses, whereas small spines are generally transient and form weaker synapses (Takumi et al., 1999; Matsuzaki et al., 2001, 2004; Yasumatsu et al., 2008). Because of these properties, large, mushroom-type spines have been hypothesized to represent physical substrates of long-term memories (i.e., memory spines), and small, thin spines may represent the capacity for adaptive experience-dependent rewiring of neuronal circuits (i.e., learning spines) (Kasai et al., 2003).

The dynamic nature of functions ascribed to the PFC suggests that prefrontal neurons are highly dependent on spine plasticity. This view is consistent with observations that PFC neurons have higher spine densities than neurons in visual or temporal cortices (Elston, 2000). Recently, our group reported selective thin spine loss in aged monkey layer III dorsolateral PFC neurons (Dumitriu et al., 2010). Furthermore, changes in thin spine morphology correlated with the ability to acquire a delayed nonmatching-tosample task (Dumitriu et al., 2010), suggesting that age-related behavioral impairments may be a function of changes in thin spine plasticity. These patterns of spine changes are suggestive of an overall reduced ability to rewire PFC circuits in aged animals, although no study has directly examined the capacity for experience-dependent spine plasticity in aging prefrontal neurons. Here we test the hypothesis that aging spines show reduced plasticity in a behavioral stress paradigm known to pro- 
duce spine remodeling (Radley et al., 2006, 2008). The data presented here provide the first evidence that experience-dependent spine plasticity mechanisms are altered by age in the PFC.

\section{Materials and Methods}

Animals and stress paradigm. The animals used were the same as those used in our previous study (Bloss et al., 2010). Male Sprague Dawley rats (Harlan) at 3, 12, and 20 months of age (i.e., young, middle-aged, and aged; $n=20-22$ per age) were single-housed in clear polycarbonate cages $(45 \times 25 \times 20 \mathrm{~cm})$ with woodchip bedding on a 12:12 light:dark cycle at $21 \pm 2^{\circ} \mathrm{C}$ and allowed standard rat chow and tap water ad libitum. Rats were allowed 1 week to acclimate to the Rockefeller University vivarium before the onset of our stress paradigm. Chronic restraint stress entailed placing rats in wire mesh restrainers at $1000 \mathrm{~h}$ and removing them at $1600 \mathrm{~h}$ daily for 21 consecutive days. To determine the transient or persistent nature of stress effects on prefrontal neurons, a recovery group was included in these studies and was allowed to rest for an additional $21 \mathrm{~d}$ in the same vivarium. All experiments were conducted in compliance with the NIH guidelines for the Care and Use of Experimental Animals and approved by the Institutional Animal Care and Use Committees at Mount Sinai School of Medicine and Rockefeller University.

Perfusions and tissue processing. Approximately $24 \mathrm{~h}$ after the last stressor, animals were overdosed with $100 \mathrm{mg} / \mathrm{kg}$ sodium pentobarbital and perfused with $1 \%$ paraformaldehyde at $50 \mathrm{ml} / \mathrm{min}$ in $0.1 \mathrm{M}$ phosphate buffer (ice-cold, $\mathrm{pH}$ 7.3) for $1 \mathrm{~min}$, then with $4 \%$ paraformaldehyde plus $0.125 \%$ glutaraldehyde in $0.1 \mathrm{M}$ phosphate buffer (ice-cold, pH 7.3) at $50 \mathrm{ml} / \mathrm{min}$ for $12 \mathrm{~min}$. Brains were removed, postfixed for $6 \mathrm{~h}$ in the paraformaldehyde-glutaraldehyde fixative, and cut into $250 \mu \mathrm{m}$ coronal sections using a Vibratome (VT1000S; Leica). Sections encompassing the entire prelimbic (PL) cortex of the medial PFC [PL anterior border, $5.16 \mathrm{~mm}$; PL posterior border, $2.52 \mathrm{~mm}$ from bregma (Paxinos and Watson, 2005)] were stored in $0.1 \mathrm{M}$ PBS at $4^{\circ} \mathrm{C}$ until ionophoretic cell filling.

Ionophoretic cell filling. Ionophoretic cell filling was performed exactly as described previously (Radley et al., 2006; Bloss et al., 2010). Approximately five or six sections per animal were available for cell filling, encompassing the entire rostral-caudal extent of the PL cortex. Sections were stained with 4,6,-diamidino-2-phenylindole for $5 \mathrm{~min}$ to identify the dorsoventral PL borders and the neocortical laminae under ultraviolet epifluorescence. Neurons in layer III of the PL cortex were impaled with a glass micropipette containing $5 \%$ Lucifer yellow (Invitrogen) in distilled water and injected at $1-6 \mathrm{nA}$ for $\sim 5$ min to completely fill all distal aspects of the dendritic tree. Slices were washed in PBS, mounted on SuperFrost Plus microscope slides (Fisher Scientific), coverslipped with FluoroMount-G (Becton Dickinson), and stored at $4^{\circ} \mathrm{C}$.

Confocal laser scanning microscopy and NeuronStudio spine morphological analysis. Using dendritic traces generated from the same neurons in our previous study (Bloss et al., 2010), concentric circles in increments of $75 \mu \mathrm{m}$ in diameter were drawn over the trace from the center of the cell soma to provide a systematic sampling of distal, intermediate, and proximal dendritic segments (Fig. $1 A, B$ ). A random subset of five to nine neurons was imaged per animal. Approximately one to four secondary or tertiary segments at 225,150 , and $75 \mu \mathrm{m}$ from the soma in the apical tree and at $75 \mu \mathrm{m}$ from the soma in the basal tree per cell were captured by laser scanning microscopy using a Zeiss 510 confocal microscope equipped with an argon laser, Zeiss $100 \times 1.4$ NA oil-immersion objec-
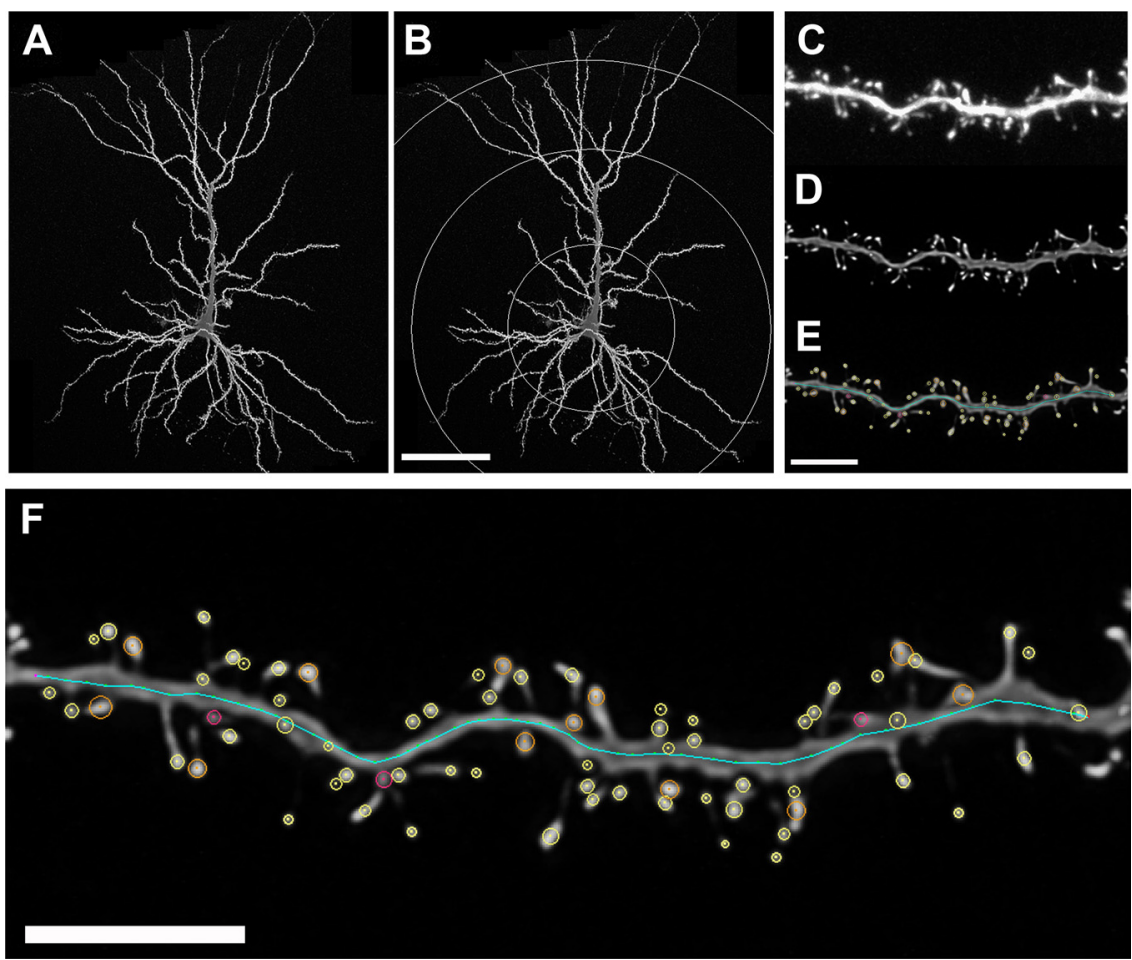

Figure 1. Spine sampling and reconstructions. $\boldsymbol{A}, \boldsymbol{B}$, Layer III PL neurons were ionophoretically labeled with Lucifer yellow $(\boldsymbol{A})$ and systematically sampled at $75 \mu \mathrm{m}$ intervals in the apical and basal tree $(\boldsymbol{B}) . \boldsymbol{C}-\boldsymbol{E}$, Raw confocal laser scanning microscopy custom-built NeuronStudio software package $(\boldsymbol{E})$. $\boldsymbol{F}$, High-resolution example of dendritic spine analysis. Circles of all colors, maximum head diameter measures; yellow circles, thin spines; orange circles, mushroom spines; pink, stubby spines. See Materials and Methods for details. Scale bars: $\boldsymbol{A}, \boldsymbol{B}$ (in $\boldsymbol{B}$ ), $75 \mu \mathrm{m} ; \boldsymbol{C}-\boldsymbol{F}, 5 \mu \mathrm{m}$.

tive, and ZEN software (Fig. 1C). To be included in the analyses, segments from dendritic branches had to satisfy the following criteria: (1) located within a depth of $100 \mu \mathrm{m}$ from the surface of the section because of the limited working distance of the objective, (2) be either parallel to or at acute angles relative to the coronal surface of the section to allow unambiguous identification of spines, and (3) segments had no overlap with other branches that would obscure visualization of spines. Conditions such as laser excitation (458 nm), fluorescence emission capture (505-530 nm), pinhole size ( 1 airy disc), and frame averaging (4 frames/ $z$-step) were held constant throughout the study. Confocal $z$-stacks were taken with a digital zoom of 3.3, a $z$-step of $0.1 \mu \mathrm{m}$, and at $512 \times 512$ pixel resolution, yielding an image with pixel dimensions of $0.05 \times 0.05 \times 0.1$ $\mu \mathrm{m}$. Z $Z$-stacks were deconvolved using AutoDeblur software (version 8.0.2) (Fig. $1 D$ ) to improve voxel resolution and reduce optical aberration along the $z$-axis, and were then imported and analyzed using custom-built NeuronStudio software (Rodriguez et al., 2006, 2008; Radley et al., 2008). Three-dimensional automated NeuronStudio analysis included spine density, spine type (e.g., mushroom, thin, stubby), and spine head diameter (Fig. $1 E$ ). Spines were classified as thin or mushroom if the ratio of their maximum head diameter to maximum neck diameter was $>1.1$. Of the spines that met this criterion, those with a maximum head diameter $<0.4 \mu \mathrm{m}$ were classified as thin spines and those with maximum spine head diameters $>0.4 \mu \mathrm{m}$ were classified as mushroom spines. Spines with a head:neck ratio $<1.1$ were classified as stubby spines. Filopodia, characterized by long, thin protrusions without a swelling at the distal tip, were encountered very rarely and were scored as thin spines. To minimize bias, all analyses were done blind to the experimental condition.

Statistical analyses. Dendritic spine parameters were initially collapsed across all dendritic distances (i.e., overall analysis), averaged for each animal, and analyzed using a one-way ANOVA followed by Bonferroni's post hoc tests (GraphPad Software). Subsequent analyses at specific dendritic distances (i.e., $225 \mu \mathrm{m}$ apical tree, $150 \mu \mathrm{m}$ apical tree, $75 \mu \mathrm{m}$ apical 
A

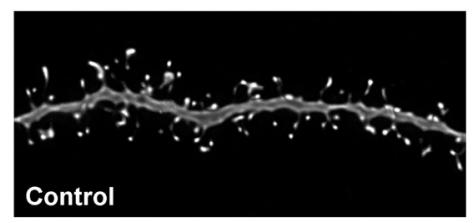

B

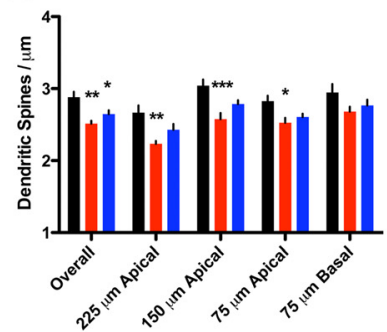

C

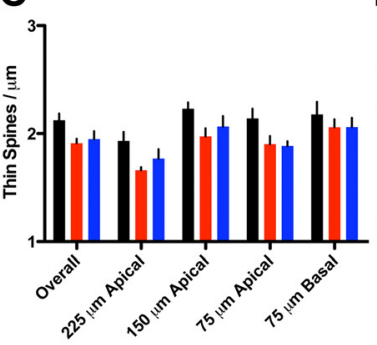

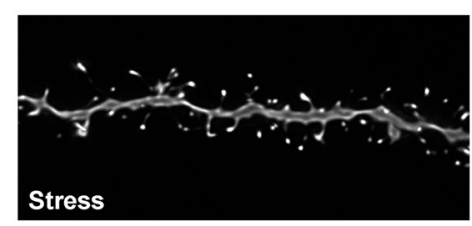

D

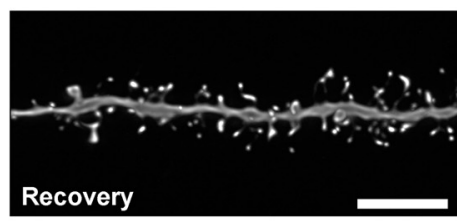

E
F

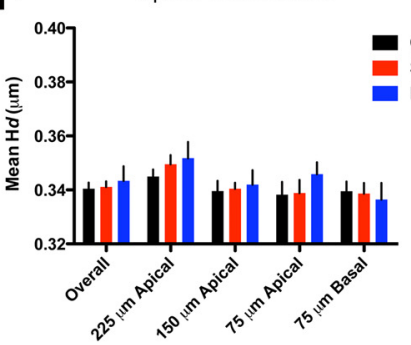

G

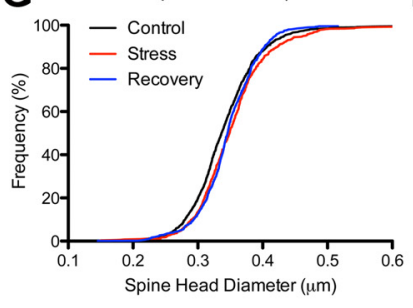

H

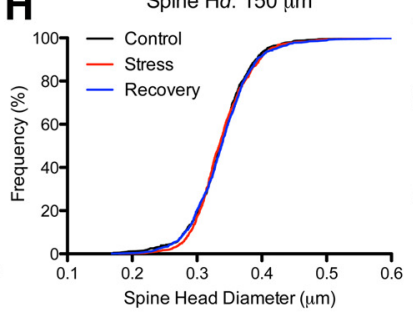

Spine Hd: 75 um Apical

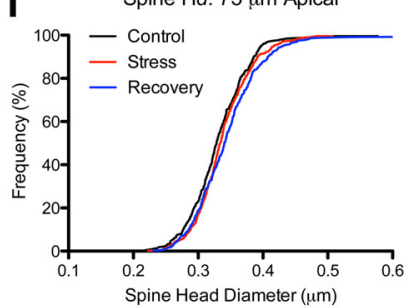

Figure 2. Stress-induced dendritic spine plasticity on neurons from young animals. $A$, Representative dendritic segments from control (left), stress (center), and recovery (right) neurons from young animals. Scale bar, $5 \mu \mathrm{m}$. B. Three weeks of stress exposure was associated with reductions in spine density throughout the apical, but not basal, tree; spine density was reduced on recovery neurons when collapsed across all dendritic distances, but not when analyzed separately. C, $\boldsymbol{D}$, Stress was associated with mild decreases in thin spine density (C), whereas mushroom spine densities were not changed by stress exposure (D).E, Stress resulted in significant decreases in stubby spine density, specifically at $150 \mu \mathrm{m}$ from the soma in the apical tree. $\boldsymbol{F}$, Stress did not alter mean spine head diameter $(\mathrm{Hd})$. $\mathbf{G}$, Individual head diameter frequencies were shifted to the right at $225 \mu \mathrm{m}$ from soma in both stress and recovery spines compared with controls. Bar graphs represent the group mean \pm SEM. $\boldsymbol{H}, \boldsymbol{I}$, Spines at 150 and $75 \mu \mathrm{m}$ were unaffected. ${ }^{*} p<0.05,{ }^{* *} p<0.005$, and ${ }^{* * *} p<0.0001$ compared with controls. See Results for details.

tree, and $75 \mu \mathrm{m}$ basal tree) were performed using mean values composed of all segments from each distance within an animal and analyzed using a two-way repeated-measures ANOVA with condition/age as a between groups factor, distance as a within-groups factor, and Bonferonni post hoc tests to probe differences between individual groups. Note that for the sake of space, overall datasets appear on the same graph as specific distance datasets, although they were analyzed differently (i.e., one-way ANOVA vs two-way ANOVA). Cumulative frequency distributions of spine head diameter were composed of all individual spines at each apical distance and analyzed with Kolmogrov-Smirnov goodness-of-fit hypothesis tests (MatLab). For all tests, $\alpha$ was set to 0.05 and Bonferroni corrections were applied where appropriate.

\section{Results}

Interaction between stress and age on dendritic spine density To determine whether age and stress interacted to regulate spine density on PL neurons, we first performed a two-way ANOVA across all groups and ages. We found a main effect of age $\left(F_{(2,112)}=\right.$ $110.02, p<0.0001)$, but not of stress condition $\left(F_{(2,112)}=1.08\right.$, $p>0.05)$, and a significant interaction $\left(F_{(4,224)}=7.474, p<\right.$ 0.0001 ) (data not shown). These results suggest that stress affected dendritic spine density in an age-dependent manner.

\section{Stress and dendritic spine plasticity in young animals}

To further analyze the effects of stressful experience on dendritic spines from young animals, we examined $\sim 46,900$ spines (Fig. $2 A$; Table 1). We found a main effect of stress condition on overall spine density $\left(F_{(2,34)}=10.00, p<0.005\right)$, with reductions in stress $(p<0.01)$ and recovery neurons $(p<0.05)$ compared with controls (Fig. $2 B$ ). When analyzed as a function of dendritic
Table 1. Summary of the number of animals, neurons, and spines included in each group

\begin{tabular}{|c|c|c|c|}
\hline Treatment & Control & Stress & Stress + recovery \\
\hline \multicolumn{4}{|l|}{ Young } \\
\hline Animals & 7 & 6 & 7 \\
\hline Neurons & $45(5-8)$ & $41(5-8)$ & $49(5-8)$ \\
\hline Spines & $17532(1915-3122)$ & $13218(1643-2521)$ & $16564(1771-2680)$ \\
\hline \multicolumn{4}{|l|}{ Middle-aged } \\
\hline Animals & 8 & 7 & 7 \\
\hline Neurons & $45(5-6)$ & $42(5-8)$ & $42(5-7)$ \\
\hline Spines & $13903(1149-2190)$ & $15243(1264-3058)$ & $12269(1457-2230)$ \\
\hline \multicolumn{4}{|l|}{ Aged } \\
\hline Animals & 7 & 8 & 7 \\
\hline Neurons & $50(5-9)$ & $52(5-8)$ & $50(6-8)$ \\
\hline Spines & $15251(1408-2534)$ & $15194(1414-2502)$ & $13287(1631-2084)$ \\
\hline
\end{tabular}

The effects of aging were determined by comparing the control animals from each age group. Numbers in parentheses represent the range of neurons and spines used within each group.

distance from the soma, two-way ANOVA revealed effects of condition $\left(F_{(2,34)}=12.92, p<0.0005\right)$ and distance $\left(F_{(3,51)}=\right.$ 17.71, $p<0.0001)$ with no interaction; stressed neurons showed reduced spine density compared with controls at 225 $(p<0.01), 150(p<0.001)$, and $75(p<0.05) \mu \mathrm{m}$ from the soma in the apical tree, but not at $75 \mu \mathrm{m}$ from the soma in the basal tree; in contrast, recovery neurons did not significantly differ from control or stress neurons at any of these distances. We concluded that stress selectively reduced dendritic spine densities on apical, but not basal, dendritic branches; furthermore, recovery from stress allowed for moderate spine regrowth in these apical domains. 

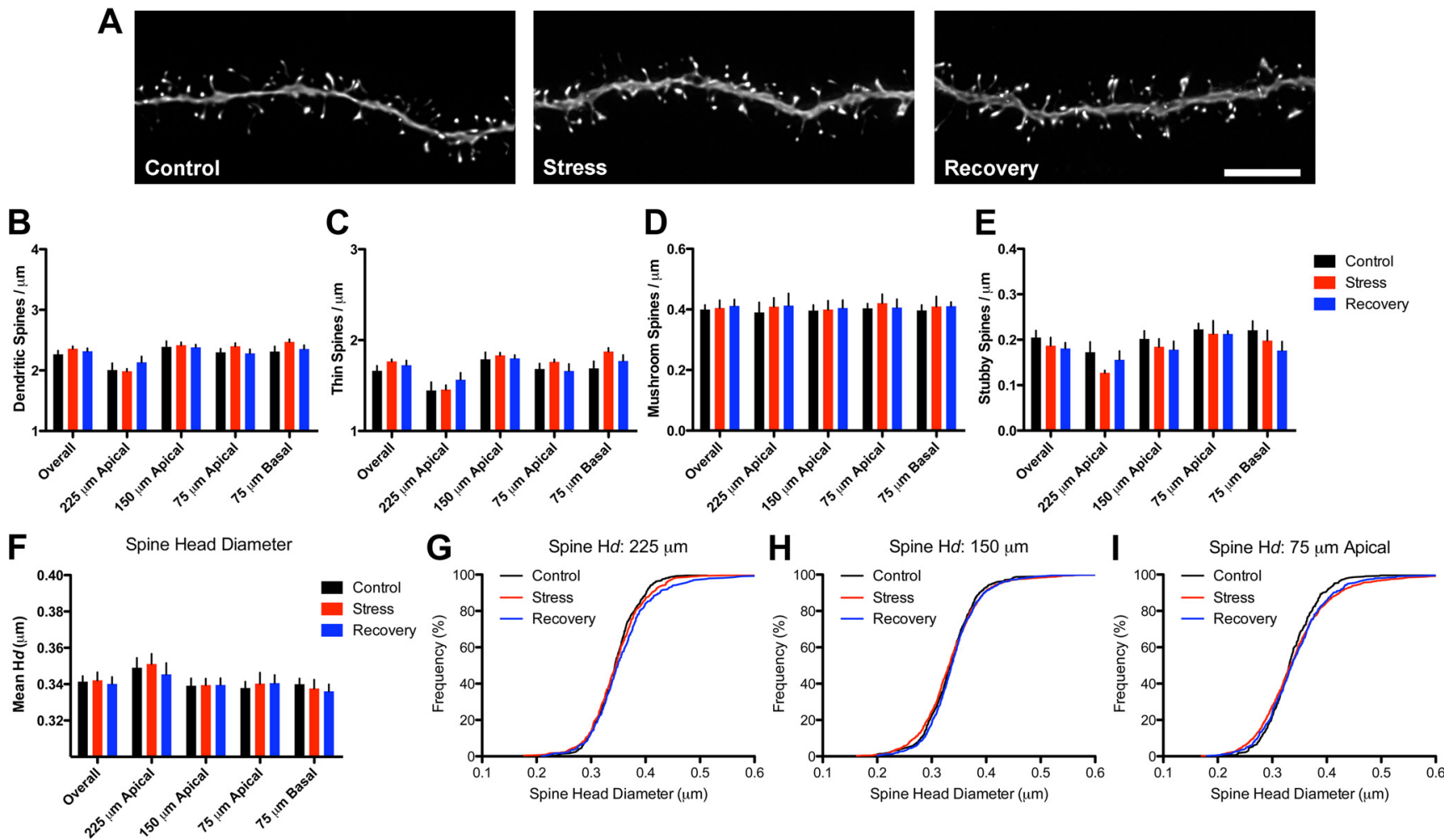

Figure 3. Spine stability on middle-aged neurons. $A$, Representative dendritic segments from control (left), stress (center), and recovery (right) neurons from middle-aged animals. Scale bar, 5 $\mu \mathrm{m} . \boldsymbol{B}$, In contrast to neurons from young animals, stress exposure did not alter spine density in middle-aged neurons. $\boldsymbol{C} \boldsymbol{F}$, Thin $(\boldsymbol{C})$, mushroom $(\boldsymbol{D})$, and stubby $(\boldsymbol{E})$ spine densities and mean spine head diameters $(\boldsymbol{F})$ were unaltered by stress or recovery. Bar graphs represent the group mean \pm SEM. $\mathbf{G}, \boldsymbol{H}$, Individual head diameter (Hd) frequency distributions at $225 \mu \mathrm{m}(\boldsymbol{G})$ from soma showed minor differences between control and recovery animals, whereas frequency distributions at $150 \mu \mathrm{m}(\boldsymbol{H})$ were significantly different between stress and recovery animals. $\boldsymbol{I}$, Spines at $75 \mu \mathrm{m}$ in the apical tree were indistinguishable by group.

As spine morphology is known to influence spine function, we next determined whether spine losses in young neurons were driven by a particular morphological population of spines (i.e., thin, mushroom, or stubby). Although an initial ANOVA showed a near-significant effect of condition on overall thin spine density ( $p=0.0576$ ) (Fig. 2C), a second analysis separating thin spine density as a function of dendritic distance revealed significant effects of condition $\left(F_{(2,34)}=4.384, p<0.05\right)$ and distance $\left(F_{(3,51)}=14.10, p<0.05\right)$ with no interaction. Post hoc tests showed that the density of thin spines was not significantly different in either stress or recovery groups at any distance compared with controls. Similar analyses of mushroom spine density revealed no overall effects of condition and we did not find any main effects of distance, condition, or an interaction when we analyzed the dendritic domains separately (Fig. 2D). There were, however, significant changes in overall stubby spine density $\left(F_{(2,34)}=5.744, p<0.05\right)$ (Fig. $\left.2 E\right)$ and subsequent analysis by distance revealed main effects of condition $\left(F_{(2,34)}=4.383, p<\right.$ $0.05)$, distance $\left(F_{(3,51)}=3.218, p<0.05\right)$, and an interaction $\left(F_{(6,102)}=2.394, p<0.05\right)$. Post hoc tests revealed a reduction in stubby spines at $150 \mu \mathrm{m}$ from the soma in the apical tree $(p<$ 0.001 ) between control and stress neurons, with no differences found between controls and recovery neurons. Collectively, these data suggest stress-induced reductions in spine density were primarily due to losses of stubby and thin spines within apical domains, whereas mushroom spines remained unaffected.

To further understand the effects of stress on spine morphology, we analyzed mean spine head diameter. We found no effect of condition on overall mean head diameter (Fig. $2 F$ ) and analysis of mean head diameter as a function of distance from soma revealed an effect of distance $\left(F_{(3,51)}=6.467, p<0.001\right)$ but not condition or condition-by-distance interaction. As mean values are rather insensitive to subtle changes, we used cumulative frequency plots to measure shifts in individual spine head diameters. We found stress caused a selective shift in head diameters at $225 \mu \mathrm{m}$ from the soma in the apical tree relative to control spines (Kolmogorov-Smirnov test, $p<0.0005)$. This effect was also present in recovery spines compared with controls (KolmogorovSmirnov test, $p<0.0005)$, with no difference between stress and recovery spines (Fig. $2 G$ ). Individual spine head diameters at 150 and $75 \mu \mathrm{m}$ did not differ between the groups (Fig. $2 \mathrm{H}, I$ ).

In summary, these data suggest stress is associated with selective decreases in dendritic spines in apical, but not basal, domains in PFC neurons from young animals. Moreover, the fact that stress causes a reduction in primarily thin and stubby spines but not mushroom spines was associated with an increase in spine head diameter at distal domains. A 3 week recovery period is associated with a modest return of spine density, but not spine morphology, in distal spines.

Stress and dendritic spine stability in aging animals

We next tested whether neurons from middle-aged animals exhibited similar stress-induced spine density and morphological changes after stress exposure. Approximately 41,400 spines were included in this age group (Fig. $3 A$; Table 1). A one-way ANOVA showed no effect of stress on spine density collapsed across all dendritic distances (Fig. 3B) and subsequent two-way ANOVA analysis of spine density as a function of dendritic distance revealed a significant effect of distance $\left(F_{(3,57)}=26.92, p<0.0001\right)$ but not of condition or a condition-by-distance interaction. Sim- 

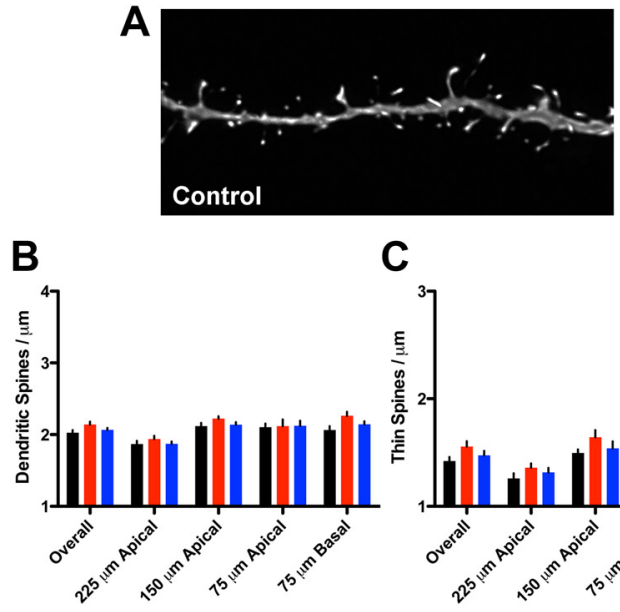

C

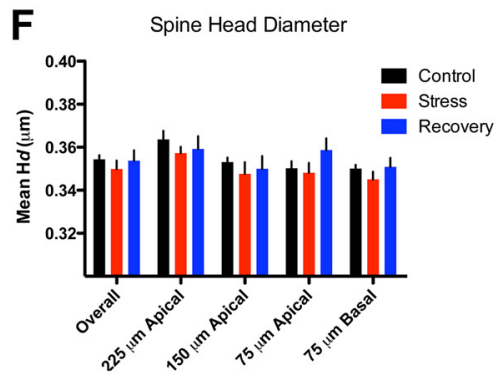

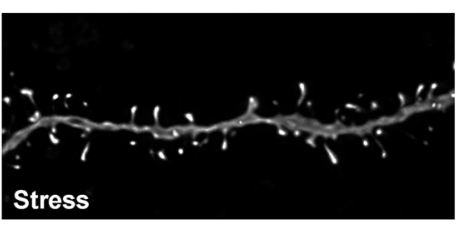

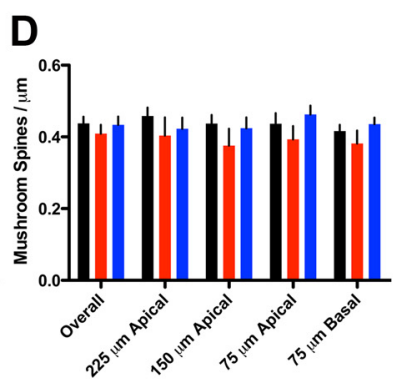

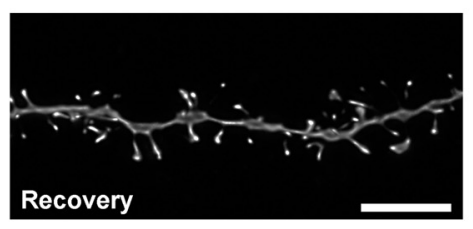

E

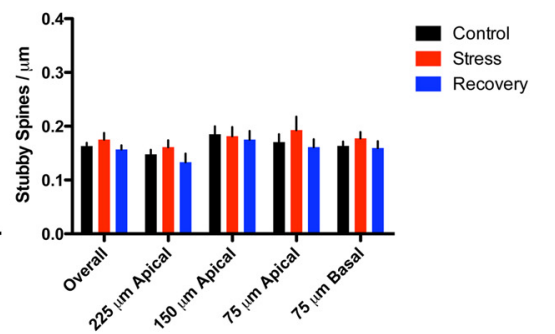

Figure 4. Spine stability on aged neurons. $\boldsymbol{A}$, Representative dendritic segments from control (left), stress (center), and recovery (right) neurons from aged animals. Scale bar, $5 \mu \boldsymbol{m}$. $\boldsymbol{B}$, Similar to middle-aged neurons, stress exposure did not alter spine density in aged neurons. $\boldsymbol{C}-\boldsymbol{F}$, Thin $(\boldsymbol{C})$, mushroom $(\boldsymbol{D})$, and stubby $(\boldsymbol{E})$ spine densities and mean spine head diameters $(\boldsymbol{F})$ were unaltered by stress or recovery. Bar graphs represent the group mean \pm SEM. $\mathbf{G}-\boldsymbol{I}$, Individual head diameter $(\mathrm{H} d)$ frequency distributions at $225(\boldsymbol{G}), 150(\boldsymbol{H})$, and $75(\boldsymbol{I}) \mu \mathrm{m}$ from soma on the apical tree were unaffected by stress or recovery.

ilarly, no effects were found on thin, mushroom, or stubby spine densities (Fig. 3C-E) when they were collapsed across all distances. Subsequent analyses separating the data by dendritic domain revealed main effects of distance on thin $\left(F_{(3,57)}=34.15\right)$ and stubby $\left(F_{(3,57)}=10.25, p<0.001\right)$ spine density, but no main effect of condition for either spine type. We did not find effects of distance or condition on mushroom spine densities. These analyses provide evidence that spine density is unaffected in response to chronic stress exposure in neurons from middle-aged animals.

To address the possibility that stress causes spine morphological remodeling in the absence of spine density changes, we examined the stress and distance effects on the mean spine head diameter and the frequency distribution of head diameters. Analysis of collapsed mean spine head diameter revealed no effect of condition and a subsequent analysis as a function of distance from the soma showed a main effect of distance $\left(F_{(3,57)}=5.617\right.$, $p<0.005)$ but not of condition or condition-by-distance interaction (Fig. $3 F$ ). Analysis of individual spine head diameters revealed no consistent pattern of morphological remodeling, although we found a minor difference among spine size distributions between control and recovery spines at $225 \mu \mathrm{m}$ (Kolmogorov-Smirnov test, $p<0.05$ ) and between stress and recovery spines at $150 \mu \mathrm{m}$ (Kolmogorov-Smirnov test, $p<0.05$ ) (Fig. $3 G-I)$. Thus, in contrast to stress-dependent changes in young animals, neurons from middle-aged animals exhibit marked rigidity in both density and morphology.

We next explored whether neurons from aged animals (i.e., 20 months) exhibited stress- or recovery-related remodeling of spines. We analyzed $\sim 43,700$ spines in this age group (Fig. $4 A$; Table 1). Similar to middle-aged animals, we found no effect of condition on overall spine density in neurons from aged animals.
Subsequent analysis as a function of distance revealed a main effect of distance on spine density $\left(F_{(3,57)}=24.62, p<0.001\right)$ but no main effect of condition or condition-by-distance interaction (Fig. $4 B$ ). Collapsed analyses of spine density by spine type (Fig. $4 C-E$ ) showed no overall effects of conditions on any spine type and subsequent two-way ANOVAs analyzing subtypes across the different dendritic domains showed effects of distance on thin $\left(F_{(3,57)}=27.59, p<0.0001\right)$ and stubby $\left(F_{(3,57)}=3.775, p<\right.$ $0.05)$ spines with neither type showing effects of condition or condition-by-distance interactions. Mushroom spine density did not differ by either distance or condition.

We next determined whether aged spines showed evidence of stress-induced spine morphological remodeling. Collapsed analysis of overall mean head diameter showed no effect of condition and analysis of spine head diameter as a function of distance using a two-way ANOVA showed a main effect of distance $\left(F_{(3,57)}=10.48, p<0.001\right)$ but no main effect of condition or condition-by-distance interaction (Fig. $4 F$ ). Analysis of individual spine head diameter distributions at 225,150 , and $75 \mu \mathrm{m}$ did not reveal any significant differences between the groups (Fig. $4 G-I)$. From these data, we conclude that, like middle-aged animals, PL neurons from aged animals failed to respond to stress exposure with changes in density or morphology.

\section{Aging-related spine loss and altered spine morphology}

We focused on whether the failure of stress-associated spine changes in the aging neurons was associated with age-related changes in spine density and/or morphology. In this analysis, we used data obtained from our control animals at each age (Fig. $5 A$; Table 1). Analysis of overall spine density revealed robust effects of age $\left(F_{(2,38)}=53.96, p<0.0001\right)$, with differences between 


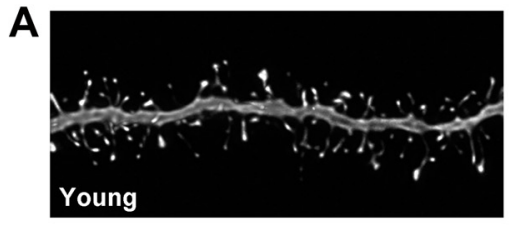

B

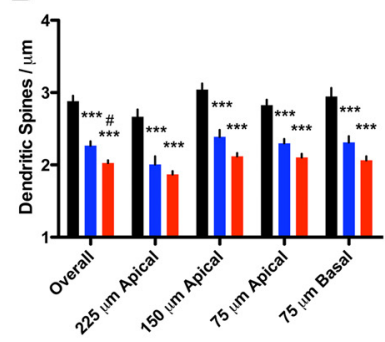

F Spine Head Diameter

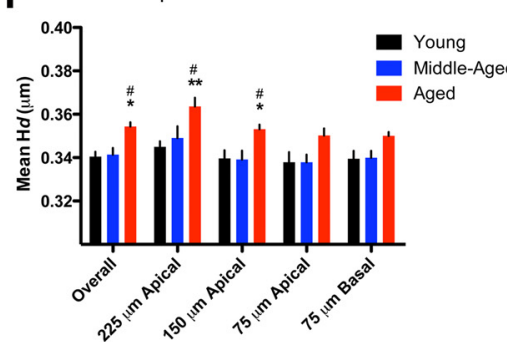

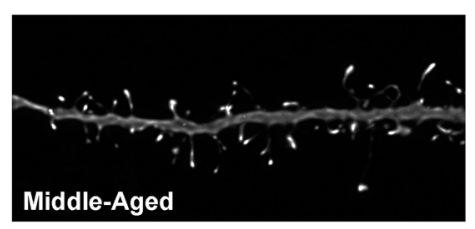

D

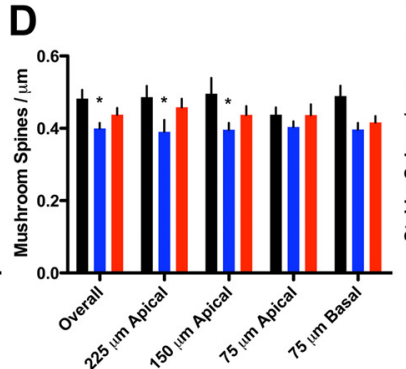

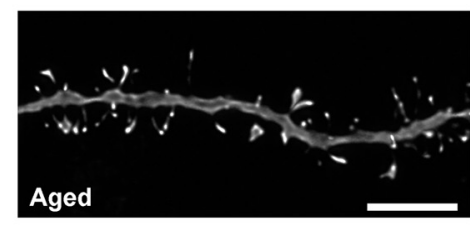

E

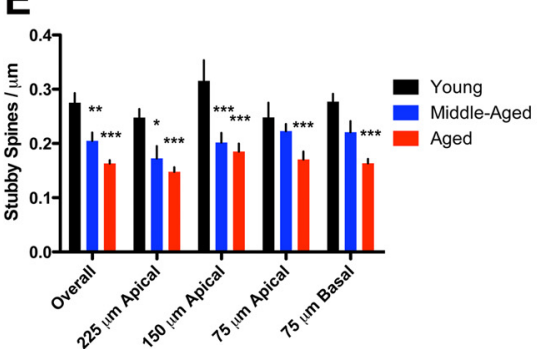

Figure 5. Changes in spine density and morphology on aging control neurons. $\boldsymbol{A}$, Representative dendritic segments from young control (left), middle-aged control (center), and aged control (right) animals. Scale bar, $5 \mu \mathrm{m}$. B, Robust decreases in spine density were found across all aging control neurons. $\boldsymbol{C}$, Thin spine densities were progressively decreased across the dendritic tree with age. $\boldsymbol{D}$, Mushroom spine densities were decreased at distal segments in middle-aged compared with young neurons, but otherwise remained stable between young and aged animals. $\boldsymbol{E}$, Like thin spines, stubby spine densities progressively decreased across the dendritic tree with age. $\boldsymbol{F}$, Aging was associated with significant increases in mean spine head diameter overall and specifically in distal apical segments of aged, but not middle-aged, animals. Bar graphs represent the group mean \pm SEM. G-I, Individual head diameter (Hd) frequency distributions were significantly shifted to the right at all apical distances in aged spines relative to both young and middle-aged spines. ${ }^{*} p<0.05,{ }^{* *} p<0.005$, and ${ }^{* * *} p<0.0001$ compared with young. $p<0.05$ compared with middle-aged. See Results for details.

young and middle-aged $(p<0.001)$, young and aged $(p<$ 0.0001 ), and middle-aged and aged ( $p<0.05$ ) animals (Fig. $5 B$ ). Subsequent two-way ANOVA analysis of spine density as a function of dendritic distance showed main effects of distance $\left(F_{(3,57)}=14.59, p<0.0001\right)$ and age $\left(F_{(2,38)}=51.7, p<0.0001\right)$ but no age-by-distance interaction. Post hoc tests revealed robust differences between young and middle-aged and between young and aged at 225, 150, and $75 \mu \mathrm{m}$ on the apical dendrites and 75 $\mu \mathrm{m}$ on the basal dendrites ( $p<0.0001$ for all analyses). Middleaged and aged spine densities did not differ at any specific distance examined. These data suggest aging is associated with widespread spine loss on layer III PFC neurons across all areas of the apical and basal dendritic tree, and contrasts with the more selective pattern of apical spine loss observed in young animals exposed to stress.

We next asked whether these age-related spine losses were from any specific spine type populations. We found overall effects of age on thin spine density $\left(F_{(2,38)}=43.91, p<0.0001\right)$ with decreases in middle-aged $(p<0.0001)$ and aged $(p<$ $0.0001)$ compared with young animals, as well as a decrease between aged compared with middle-aged animals $(p<0.05)$ (Fig. $5 C)$. Two-way ANOVA to determine whether thin spines were lost across the entire dendritic tree found main effects of distance $\left(F_{(3,57)}=14.8, p<0.0001\right)$ and age $\left(F_{(2,38)}=40.59, p<0.0001\right)$ but no age-by-distance interaction; these data reveal differences in thin spine density observed across all dendritic domains between young and middle-aged ( $p<0.001$ for all distances) and between young and aged ( $p<0.001$ for all distances) animals. In addition, we found a significant difference between middle-aged and aged animals at $150 \mu \mathrm{m}(p<0.05)$. Mushroom spine density analysis (Fig. $5 D)$ revealed an overall effect of age $\left(F_{(2,38)}=4.837\right.$, $p<0.05)$, with a significant difference between young and middle-aged $(p<0.05)$, but not young and aged, neurons. Twoway ANOVA of mushroom spines by dendritic distance showed no significant effects of distance but an effect of age $\left(F_{(2,38)}=\right.$ 4.991, $p<0.05)$ such that neurons from middle-aged animals had lower mushroom spine densities at 225 and $150 \mu \mathrm{m}(p<$ 0.05 for both) in the apical tree compared with neurons from young animals. Collapsed analysis of stubby spines (Fig. $5 E$ ) revealed an overall effect of age $\left(F_{(2,38)}=15.31, p<0.0001\right)$, with differences between young and middle-aged $(p<0.001)$ and young and aged $(p<0.0001)$ animals, but not between middleaged and aged animals. Subsequent two-way ANOVA analysis of stubby spine density showed main effects of distance $\left(F_{(3,57)}=\right.$ 5.098, $p<0.001)$ and age $\left(F_{(2,38)}=11.85, p<0.001\right)$ with no age-by-distance interaction; post hoc tests revealed stubby spine density was reduced at $225(p<0.05)$ and $150 \mu \mathrm{m}(p<0.001)$ from the soma in the apical tree in middle-aged compared with young animals, and across all distances in aged compared with young animals $(225 \mu \mathrm{m}, p<0.01 ; 150 \mu \mathrm{m}, p<0.001 ; 75 \mu \mathrm{m}$ apical, $p<0.05 ; 75 \mu \mathrm{m}$ basal, $p<0.001)$. No differences in stubby spine density were observed between middle-aged and aged animals at any distances. Together, these data provide evidence that age-related spine losses were primarily due to thin and stubby spines; mushroom spines, in contrast, remained relatively stable across all ages. Because stubby spines did not differ between 
neurons from middle-aged and aged animals, our data provide evidence that the progressive nature of spine loss between 12 and 20 months is best explained by a further loss of thin spines.

Finally, we examined whether morphological changes occurred in spine head diameter as a result of the aging process. We found a main effect of age on collapsed mean spine head diameter $\left(F_{(2,38)}=8.820, p<0.05\right)$ with differences between young and aged $(p<0.01)$ and between middle-aged and aged $(p<0.01)$ spines (Fig. $5 F$ ). Subsequent two-way ANOVA of mean spine head diameter by distance revealed main effects of distance $\left(F_{(3,57)}=6.592, p<0.001\right)$ and age $\left(F_{(2,38)}=9.393, p<0.005\right)$ in spine head diameter, but no age-by-distance interaction. We found increases in mean head diameter in aged spines at 225 and $150 \mu \mathrm{m}$ compared with young $(p<0.001$ and $p<0.05$, respectively) and between aged and middle-aged ( $p<0.05$ for both distances) spines. We next tested for potential age-related changes in the frequency of individual spines by head diameter; a clear difference was revealed at 225,150 , and $75 \mu \mathrm{m}$ from soma in the apical tree between young and aged spines (KolmogorovSmirnov tests: $225 \mu \mathrm{m}, p<0.0001 ; 150 \mu \mathrm{m}, p<0.0001 ; 75 \mu \mathrm{m}$, $p<0.0001$ ) (Fig. $5 G-I$ ). We also found differences in frequency distribution between middle-aged and aged spines (KolmogorovSmirnov tests: $225 \mu \mathrm{m}, p<0.005 ; 75 \mu \mathrm{m}, p<0.0001$ ), suggesting the shift in head diameter distribution primarily occurred between 12 and 20 months. These data corroborate the notion that the spine loss between middle-aged and aged animals is comprised principally of thin spines.

Together, these results suggest spine loss with aging is characterized by a selective and progressive decrease of thin and stubby spines while large, mushroom-type spines remain stable. As a result, these processes are reflected by an increase in mean head diameter in aged spines, which is not yet evident in middle-aged spines. Last, these results provide a crucial link between agerelated changes in spine morphology and reductions in the capacity to undergo experience-dependent spine plasticity.

\section{Discussion}

Here, we used a chronic stress paradigm to determine how spine plasticity was affected by aging in PFC neurons. Our systematic sampling procedure and automated spine analysis allowed us to sample $>130,000$ dendritic spines in an unbiased fashion. Our major findings are that changes in spine density and morphology with stress in young rats are absent in middle-aged and aged rats, and that loss of spine plasticity occurs alongside age-related declines in spine density and shifts in spine morphology. The present study provides the first direct evidence that aging modulates the capacity for experience-dependent spine plasticity in PFC neurons.

\section{Stress-induced spine alterations in young animals}

These data corroborate previous studies showing that stress reduces spine density on PL neurons (Radley et al., 2006, 2008; Liu and Aghajanian, 2008; Hains et al., 2009). As in earlier reports, stress selectively reduced apical, but not basal, dendritic spines (Radley et al., 2006; Dias-Ferreira et al., 2009; Hains et al., 2009). Since spines exhibit constant turnover in neocortex (Bhatt et al., 2009), we conclude that stress-induced reductions of spine density are the result of either decreased rates of spine formation or increased rates of spine elimination. Future studies using in vivo repeated spine sampling will be required to differentiate these two possibilities.

To our knowledge, the current study is the first to test whether a stress-free recovery period results in normalization of spine densities on PL neurons. In contrast to previous reports demonstrating dendritic recovery from stress exposure (Radley et al., 2005; Goldwater et al., 2009; Bloss et al., 2010), our observations suggest stress-induced changes at the level of the spine may persist through a 3 week recovery period. However, the effect of stress plus recovery on spine density was not seen when analyzed by dendritic distance, and total estimated spine number per apical dendritic tree was not different between control and recovery animals (data not shown). Together with previous studies (Radley et al., 2005; Goldwater et al., 2009; Bloss et al., 2010), the current findings imply a high level of adaptive structural plasticity in neurons that may be functionally relevant to recoveryrelated behavioral normalization (Sousa et al., 2000; Liston et al., 2009).

In addition to spine loss, we report here that stress causes morphological changes at distal apical dendritic spines in young animals. Although we found no change in mean spine head diameter with stress or recovery, the frequency distribution of spine size on apical dendrites $225 \mu \mathrm{m}$ from the soma was shifted to the right, indicating increased head diameters in stress and recovery animals. These observations contrast with a recent report, which found stress caused a shift toward smaller spines (Radley et al., 2008). This discrepancy could be explained by several experimental differences, as the imaging parameters and random sampling scheme differed between the two studies, or the random sample of neurons included in the current analysis might be composed of different subpopulations of layer III PL neurons. In support of this last possibility, experience-dependent spine changes are celltype-dependent even within neurons located in the same layer (Holtmaat et al., 2006) and the effects of stress on neuronal remodeling in layer III infralimbic cortical neurons are circuitspecific (Shansky et al., 2009). Future studies will be needed to resolve whether stress-induced changes in PL spine morphology are a function of circuit, morphological, physiological, and/or molecular characteristics.

\section{Stress fails to alter spines in aging animals}

We previously reported that aging animals undergo stressinduced weight loss, adrenal gland hypertrophy, and reductions of PL dendritic complexity (Bloss et al., 2010). Furthermore, a substantial amount of literature suggests that the effects of stress on cognitive, neuroendocrine, biochemical, and cellular measures are exacerbated in aged animals (for review, see Sapolsky et al., 1986; Sapolsky, 1999; Lupien et al., 2009; Juster et al., 2010). Based on this literature, we anticipated enhanced spine changes in response to stress in aging animals. In contrast, the major finding here is that middle-aged and aging animals showed minimal evidence of spine remodeling in response to stress. At both ages, repeated stress failed to alter spine density and produced minimal shifts in spine morphology. These results provide strong evidence that spines on layer III PFC neurons in aged animals exhibit a remarkable stability and/or rigidity.

Although the techniques used here allow only a temporal snapshot of spines, we conclude that the failure of stress exposure to induce spine loss is evidence that aging has profound effects on spine dynamics in PFC. Alternatively, it remains possible that aging selectively inhibits stress-dependent spine plasticity while leaving other forms of spine plasticity in PFC intact. However, our interpretation is consistent with in vivo two-photon data that demonstrate rates of spine turnover decrease and spines become progressively more stable over time (i.e., months) in mouse neocortex (Grutzendler et al., 2002; Holtmaat et al., 2005; Yang et al., 2009). 


\section{Age-related changes in spines accompany reduced plasticity} Comparisons between our young and aging control rats revealed progressive age-related reductions in spine density, including $\sim 20 \%$ loss in middle-aged and $\sim 30 \%$ loss in aged animals. In contrast to the selective spine remodeling in response to stress, age-related spine loss was readily apparent in distal, intermediate, and proximal dendritic branches in the apical and basal tree. These data corroborate previous observations from aging rat, nonhuman primate, and human studies of prefrontal cortical spines (Jacobs et al., 1997; Duan et al., 2003; Hao et al., 2007; Brennan et al., 2009; Dumitriu et al., 2010), suggesting spine loss is a conserved consequence of aging in the PFC.

Furthermore, our analysis revealed that the majority of spines susceptible to aging were characterized as thin and stubby spines, whereas mushroom spines were mostly resistant to aging. To our knowledge, this is the first report of any morphological pattern of spine vulnerability (or resilience) in the aging rat neocortex. As the vast majority of spines on PFC neurons are thin spines, in absolute terms our data support the hypothesis that the most vulnerable spine population to aging is thin, small spines. These data are similar, but not identical, to the patterns seen in the aging monkey dorsolateral PFC where thin spines are selectively lost while larger, mushroom spines remain (Hao et al., 2007; Dumitriu et al., 2010). Furthermore, our findings that mushroom spines display marked stability are consistent with two-photon studies showing that the large majority of stable, persistent spines in vivo are mushroom-type spines (Trachtenberg et al., 2002; Holtmaat et al., 2005).

Quantitative analysis of spine morphology revealed a significant overall increase in mean spine head diameter from aged animals relative to spines from young and middle-aged animals. This effect is most significant in distal segments of the apical tree, but is also evident in proximal apical and basal segments. Cumulative frequency plots also demonstrate that spines from aged, but not middle-aged, animals are shifted toward those bearing larger head diameters. Because of the tight relationship between spine morphology and synaptic glutamate receptor content (Takumi et al., 1999; Matsuzaki et al., 2001), it is likely these age-related morphological changes are associated with a shift toward larger synapses predominantly expressing AMPA receptors and a concomitant shift away from small, NMDA receptor-dominated synapses.

\section{Functional implications}

Previous reports observed impairments on working memory tasks as early as middle-age in animals and humans (Moore et al., 2006; Bizon et al., 2009; Salthouse, 2009), suggesting the impairment in spine plasticity seen in middle-age animals may be functionally relevant. Future studies should focus on whether variability in spine dynamics explains individual differences in cognitive performance commonly seen across aging animal models. Along these lines, hypothalamic-pituitaryadrenal axis activity has been linked to individual differences in age-related cognitive decline (Sapolsky et al., 1986; Issa et al., 1990; Lupien et al., 1998; Bizon et al., 2001) and could potentially mediate or modulate age-related changes in synapse dynamics (DeKosky et al., 1984).

The present data contrast with studies of aging rat and monkey hippocampus, where synaptic density remains intact. For example, axospinous synapse number appears unchanged in aged dentate gyrus (Geinisman et al., 1986; Hara et al., 2010) and CA1 (Geinisman et al., 2004), though there is evidence to suggest a selective vulnerability of large, perforated synapses in both re- gions that correlates with behavioral performance (Geinisman et al., 1986; Nicholson et al., 2004). Such a differential vulnerability between large spines in hippocampus and small spines in PFC may pose a considerable challenge in designing therapeutics to rescue age-related cognitive decline.

Together, these experiments provide evidence that agerelated dendritic spine alterations are associated with diminished experience-dependent neocortical spine plasticity. Our data support a model in which dendritic spines become progressively less plastic with age in PFC. These changes might reflect an age-related pathological change short of neurodegeneration or, alternatively, an evolutionary program that favors neuronal circuit stability over plasticity as we age. Either way, understanding how this process occurs, and which aspects may be amenable to treatment, should be a major goal for future studies aimed at ameliorating changes in neuronal plasticity and cognition during aging.

\section{References}

Bhatt DH, Zhang S, Gan WB (2009) Dendritic spine dynamics. Annu Rev Physiol 71:261-282.

Bizon JL, Helm KA, Han JS, Chun HJ, Pucilowska J, Lund PK, Gallagher M (2001) Hypothalamic-pituitary-adrenal axis function and corticosterone receptor expression in behaviourally characterized young and aged Long-Evans rats. Eur J Neurosci 14:1739-1751.

Bizon JL, LaSarge CL, Montgomery KS, McDermott AN, Setlow B, Griffith WH (2009) Spatial reference and working memory across the lifespan of male Fischer 344 rats. Neurobiol Aging 30:646-655.

Bloss EB, Janssen WG, McEwen BS, Morrison JH (2010) Interactive effects of stress and aging on structural plasticity in the prefrontal cortex. J Neurosci 30:6726-6731.

Brennan AR, Yuan P, Dickstein DL, Rocher AB, Hof PR, Manji H, Arnsten AF (2009) Protein kinase C activity is associated with prefrontal cortical decline in aging. Neurobiol Aging 30:782-792.

Burke SN, Barnes CA (2006) Neural plasticity in the ageing brain. Nat Rev Neurosci 7:30-40.

DeKosky ST, Scheff SW, Cotman CW (1984) Elevated corticosterone levels: a possible cause of reduced axon sprouting in aged animals. Neuroendocrinology 38:33-38.

Dias-Ferreira E, Sousa JC, Melo I, Morgado P, Mesquita AR, Cerqueira JJ, Costa RM, Sousa N (2009) Chronic stress causes frontostriatal reorganization and affects decision-making. Science 325:621-625.

Duan H, Wearne SL, Rocher AB, Macedo A, Morrison JH, Hof PR (2003) Age-related dendritic and spine changes in corticocortically projecting neurons in macaque monkeys. Cereb Cortex 13:950-961.

Dumitriu D, Hao J, Hara Y, Kaufmann J, Janssen WG, Lou W, Rapp PR, Morrison JH (2010) Selective changes in thin spine density and morphology in monkey prefrontal cortex correlate with aging-related cognitive impairment. J Neurosci 30:7507-7515.

Elston GN (2000) Pyramidal cells of the frontal lobe: all the more spinous to think with. J Neurosci 20:RC95.

Gallagher M, Rapp PR (1997) The use of animal models to study the effects of aging on cognition. Annu Rev Psychol 48:339-370.

Geinisman Y, de Toledo-Morrell L, Morrell F (1986) Loss of perforated synapses in the dentate gyrus: morphological substrate of memory deficit in aged rats. Proc Natl Acad Sci U S A 83:3027-3031.

Geinisman Y, Ganeshina O, Yoshida R, Berry RW, Disterhoft JF, Gallagher M (2004) Aging, spatial learning, and total synapse number in the rat CAl stratum radiatum. Neurobiol Aging 25:407-416.

Goldwater DS, Pavlides C, Hunter RG, Bloss EB, Hof PR, McEwen BS, Morrison JH (2009) Structural and functional alterations to rat medial prefrontal cortex following chronic restraint stress and recovery. Neuroscience 164:798-808.

Grady CL (2008) Cognitive neuroscience of aging. Ann N Y Acad Sci 1124:127-144.

Grutzendler J, Kasthuri N, Gan WB (2002) Long-term dendritic spine stability in the adult cortex. Nature 420:812-816.

Hains AB, Vu MA, Maciejewski PK, van Dyck CH, Gottron M, Arnsten AF (2009) Inhibition of protein kinase $C$ signaling protects prefrontal cortex 
dendritic spines and cognition from the effects of chronic stress. Proc Natl Acad Sci U S A 160:17957-17962.

Hao J, Rapp PR, Janssen WG, Lou W, Lasley BL, Hof PR, Morrison JH (2007) Interactive effects of age and estrogen on cognition and pyramidal neurons in monkey prefrontal cortex. Proc Natl Acad Sci U S A 104: $11465-11470$

Hara Y, Park CS, Janssen WG, Roberts MT, Morrison JH, Rapp PR (2010) Synaptic correlates of memory and menopause in the hippocampal dentate gyrus in rhesus monkeys. Neurobiol Aging. Advance online publication. Retrieved February 15, 2011. doi:10.1016/j.neurobiolaging.2010.09.014.

Harris KM, Stevens JK (1989) Dendritic spines of CA 1 pyramidal cells in the rat hippocampus: serial electron microscopy with reference to their biophysical characteristics. J Neurosci 9:2982-2997.

Hof PR, Morrison JH (2004) The aging brain: morphomolecular senescence of cortical circuits. Trends Neurosci 27:607-613.

Holtmaat A, Svoboda K (2009) Experience-dependent structural synaptic plasticity in the mammalian brain. Nat Rev Neurosci 10:647-658.

Holtmaat AJ, Trachtenberg JT, Wilbrecht L, Shepherd GM, Zhang X, Knott GW, Svoboda K (2005) Transient and persistent dendritic spines in the neocortex in vivo. Neuron 45:279-291.

Holtmaat A, Wilbrecht L, Knott GW, Welker E, Svoboda K (2006) Experience-dependent and cell-type-specific spine growth in the neocortex. Nature 441:979-983.

Issa AM, Rowe W, Gauthier S, Meaney MJ (1990) Hypothalamic-pituitaryadrenal activity in aged, cognitively impaired and cognitively unimpaired rats. J Neurosci 10:3247-3254.

Jacobs B, Driscoll L, Schall M (1997) Life-span dendritic and spine changes in areas 10 and 18 of human cortex: a quantitative Golgi study. J Comp Neurol 386:661-680.

Juster RP, McEwen BS, Lupien SJ (2010) Allostatic load biomarkers of chronic stress and impact on health and cognition. Neurosci Biobehav Rev 35:2-16.

Kasai H, Matsuzaki M, Noguchi J, Yasumatsu N, Nakahara H (2003) Structure-stability-function relationships of dendritic spines. Trends Neurosci 26:360-368.

Liston C, McEwen BS, Casey BJ (2009) Psychosocial stress reversibly disrupts prefrontal processing and attentional control. Proc Natl Acad Sci U S A 106:912-917.

Liu RJ, Aghajanian GK (2008) Stress blunts serotonin- and hypocretinevoked EPSCs in prefrontal cortex: role of corticosterone-mediated apical dendritic atrophy. Proc Natl Acad Sci U S A 105:359-364.

Lupien SJ, de Leon M, de Santi S, Convit A, Tarshish C, Nair NP, Thakur M, McEwen BS, Hauger RL, Meaney MJ (1998) Cortisol levels during human aging predict hippocampal atrophy and memory deficits. Nat Neurosci 1:69-73.

Lupien SJ, McEwen BS, Gunnar MR, Heim C (2009) Effects of stress throughout the lifespan on the brain, behaviour and cognition. Nat Rev Neurosci 10:434-445.

Matsuzaki M, Ellis-Davies GC, Nemoto T, Miyashita Y, Iino M, Kasai H (2001) Dendritic spine geometry is critical for AMPA receptor expression in hippocampal CA1 pyramidal neurons. Nat Neurosci 4:1086-1092.

Matsuzaki M, Honkura N, Ellis-Davies GC, Kasai H (2004) Structural basis of long-term potentiation in single dendritic spines. Nature 429:761-766.

Miller EK (2000) The prefrontal cortex and cognitive control. Nat Rev Neurosci 1:59-65.

Moore TL, Killiany RJ, Herndon JG, Rosene DL, Moss MB (2006) Executive system dysfunction occurs as early as middle-age in the rhesus monkey. Neurobiol Aging 27:1484-1493.

Nicholson DA, Yoshida R, Berry RW, Gallagher M, Geinisman Y (2004) Reduction in size of perforated postsynaptic densities in hippocampal axospinous synapses and age-related spatial learning impairments. J Neurosci 24:7648-7653.

Okamoto K, Nagai T, Miyawaki A, Hayashi Y (2004) Rapid and persistent modulation of actin dynamics regulates postsynaptic reorganization underlying bidirectional plasticity. Nat Neurosci 7:1104-1112.

Paxinos G, Watson C (2005) The rat brain in stereotaxic coordinates, 5th edition. San Diego: Elsevier.

Peters A, Kaiserman-Abramof IR (1970) The small pyramidal neuron of the rat cerebral cortex: the perikaryon, dendrites and spines. Am J Anat 127:321-355.

Radley JJ, Rocher AB, Janssen WG, Hof PR, McEwen BS, Morrison JH (2005) Reversibility of apical dendritic retraction in the rat medial prefrontal cortex following repeated stress. Exp Neurol 196:199-203.

Radley JJ, Rocher AB, Miller M, Janssen WG, Liston C, Hof PR, McEwen BS, Morrison JH (2006) Repeated stress induces dendritic spine loss in the rat medial prefrontal cortex. Cereb Cortex 16:313-320.

Radley JJ, Rocher AB, Rodriguez A, Ehlenberger DB, Dammann M, McEwen BS, Morrison JH, Wearne SL, Hof PR (2008) Repeated stress alters dendritic spine morphology in the rat medial prefrontal cortex. J Comp Neurol 507:1141-1150.

Rodriguez A, Ehlenberger DB, Hof PR, Wearne SL (2006) Rayburst sampling, an algorithm for automated three-dimensional shape analysis from laser scanning microscopy images. Nat Protoc 1:2152-2161.

Rodriguez A, Ehlenberger DB, Dickstein DL, Hof PR, Wearne SL (2008) Automated three-dimensional detection and shape classification of dendritic spines from fluorescence microscopy images. PLoS One 3:e1997.

Salthouse TA (2009) When does age-related cognitive decline begin? Neurobiol Aging 30:507-514.

Sapolsky RM (1999) Glucocorticoids, stress, and their adverse neurological effects: relevance to aging. Exp Gerontol 34:721-732.

Sapolsky RM, Krey LC, McEwen BS (1986) The neuroendocrinology of stress and aging: the glucocorticoid cascade hypothesis. Endocr Rev 7:284-301.

Shansky RM, Hamo C, Hof PR, McEwen BS, Morrison JH (2009) Stressinduced dendritic remodeling in the prefrontal cortex is circuit specific. Cereb Cortex 19:2479-2484.

Sousa N, Lukoyanov NV, Madeira MD, Almeida OF, Paula-Barbosa MM (2000) Reorganization of the morphology of hippocampal neurites and synapses after stress-induced damage correlates with behavioral improvement. Neuroscience 97:253-266.

Takumi Y, Ramirez-León V, Laake P, Rinvik E, Ottersen OP (1999) Different modes of expression of AMPA and NMDA receptors in hippocampal synapses. Nat Neurosci 2:618-624.

Trachtenberg JT, Chen BE, Knott GW, Feng G, Sanes JR, Welker E, Svoboda $\mathrm{K}$ (2002) Long-term in vivo imaging of experience-dependent synaptic plasticity in adult cortex. Nature 420:788-794.

Yang G, Pan F, Gan WB (2009) Stably maintained dendritic spines are associated with lifelong memories. Nature 462:920-924.

Yasumatsu N, Matsuzaki M, Miyazaki T, Noguchi J, Kasai H (2008) Principles of long-term dynamics of dendritic spines. J Neurosci 28:13592-13608.

Zhou Q, Homma KJ, Poo MM (2004) Shrinkage of dendritic spines associated with long-term depression of hippocampal synapses. Neuron 44: 749-757. 